Forum $2020 \cdot 35: 263$

https://doi.org/10.1007/s12312-020-00813-z

Online publiziert: 14. Juli 2020

(c) Springer Medizin Verlag $\mathrm{GmbH}$, ein Teil von

Springer Nature 2020

Deutsche Krebsgesellschaft e.V.

Berlin, Deutschland

\title{
Professor Dr. Thomas Seufferlein ist neuer Präsident der Deutschen Krebsgesellschaft
}

kontinuierliche Verbesserung der Krebsversorgung ermöglicht. Für diese Ziele setzt sich die Deutsche Krebsgesellschaft seit vielen Jahren und sehr erfolgreich ein, nicht nur im Austausch mit Fachexperten und -expertinnen, sondern auch in der Diskussion mit Entscheidern aus Politik und Gesundheitswesen, unter anderem im Nationalen Krebsplan und der Nationalen Dekade gegen Krebs. Ich freue mich sehr, gemeinsam mit den anderen Vorstandsmitgliedern, allen Sektionen der DKG und unseren Partnern, der Deutschen Krebshilfe und dem Deutschen Krebsforschungszentrum, weiter an der Umsetzung dieser Ziele arbeiten zu dürfen“, so Seufferlein.

Der Vorstand der DKG wird alle zwei Jahre gewählt und setzt sich nun wie folgt zusammen:

\section{Präsident:}

- Prof. Dr. Thomas Seufferlein (Ulm) die Gastroenterologie. Neben seiner ärztlichen Tätigkeit engagiert er sich seit vielen Jahren in der DKG, unter anderem als Vizepräsident im Vorstand, als Vorsitzender der Zertifizierungskommission der DKG-zertifizierten Darmkrebszentren, als Sprecher der DKG im Lenkungsausschuss des Leitlinienprogramms Onkologie und als Mitglied der Leitgruppen „Kolorektales Karzinom“ und „Pankreaskarzinom “ in der Arbeitsgemeinschaft Internistische Onkologie.

„Gerade in der Krebsmedizin verzeichnen wir einen rasanten Zuwachs an medizinischen Erkenntnissen. Darauf müssen wir schnell reagieren, beispielsweise mit qualitativ hochwertigen onkologischen Leitlinien, die regelmäßig aktualisiert werden, und mit einem interdisziplinären Qualitätsmanagement, das eine
Weitere Vorstandsmitglieder:

- Prof. Dr. Olaf Ortmann (Regensburg)

- Prof. Dr. Anja Mehnert-Theuerkauf (Leipzig)

- Prof. Dr. Ullrich Graeven (Mönchengladbach)

- Prof. Dr. Frederik Wenz (Freiburg)

Kongresspräsident des DKK 2022:

- Prof. Dr. Michael Ghadimi (Göttingen)

Vorstandsmitglieder der DKG-Sektion A (repräsentieren die Landeskrebsgesellschaften):

- Prof. Dr. Petra Feyer (Berlin)

- Prof. Dr. Christian Jackisch

(Offenbach)
Vorstandmitglieder der DKG-Sektion B (repräsentieren die Arbeitsgemeinschaften und interdisziplinären Studiengruppen der DKG):

- Prof. Dr. Rainer Engers (Neuss) (Wahl bereits im Vorjahr)

- Prof. Dr. Andrea Tannapfel (Bochum)

Vorstandsmitglieder der DKG-Sektion

C (fördernde Mitglieder):

- Dr. Michael Hanske (München)

- PD Dr. Georg Isbary (GrenzachWyhlen)

DKG-Generalsekretär:

- Dr. Johannes Bruns (Berlin)

\section{Korrespondenzadresse}

Katrin Mugele

Pressestelle der Deutschen

Krebsgesellschaft e.V.

mugele@krebsgesellschaft.de

Angelina Gromes

gromes@krebsgesellschaft.de 